ISSN 1997-5902

\title{
Étude de la durabilité économique et environnementale de la production de manioc sur ferralsols
}

\author{
Kouadio K.K.H..1,3, Bakayoko S.,2,3, Soro D.2,3, Ettien D.J.B.1, 3, Yoboue K. E1 \\ 1 Université Félix Houphouët-Boigny, UFR des Sciences de la Terre et des Ressources Minières (STRM), Département \\ des Sciences du Sol, 22 BP 582 Abidjan 22, Côte d'Ivoire \\ 2Université Jean Lorougnon Guédé, Département des Sciences de la Terre, 02 BP 150 Daloa 02, Côte d'Ivoire \\ ${ }^{3}$ Centre Suisse de Recherches Scientifiques en Côte d'Ivoire (CSRS), 01 BP 1303 Abidjan 01 \\ *Corresponding author email: hippolyte kouadio@yahoo.fr
}

Original submitted in on $4^{\text {th }}$ February 2014. Published online at www.m.elewa.org on 30thJune 2014. http://dx.doi.org/10.4314/jab.v78i1.5

\begin{abstract}
RESUME
Objectif : Évaluer les composantes de la durabilité des systèmes de culture à base de manioc qui s'appuient sur la culture en couloirs.

Méthodologie et résultats : l'étude a consisté à cultiver en association manioc améliorée (Yavo) / soja vert (Mung Bean) / Gliricidia sepium avec une fumure de fond (engrais NPK [20:36:36] ou la fiente de poule [10 t/ha]) en comparaison au système traditionnel composé d'une variété locale de manioc et d'arachide en culture associée sur ferralsols. La somme des nouveaux facteurs de production de manioc a permis une augmentation significative du rendement alimentaire. En plus de la production de soja, de matière organique et de bois de chauffe, le rendement en tubercules frais de la variété introduite Yavo de 40 t/ha comparé à 13 t/ha dans le système traditionnel a été mesuré. L'architecture végétale des nouveaux systèmes a favorisé une couverture permanente du sol. Même s'ils ont induit une augmentation du temps de travail, les nouveaux systèmes ont été en faveur des femmes concernant la réduction des charges au champ. Ils se sont aussi montrés viables pour un approvisionnement en nourriture, une production durable des cultures et une amélioration du revenu agricole.
\end{abstract}

Conclusion et application : Dans l'ensemble, les systèmes testés ont été plus durables dans leurs composantes agronomiques, économiques, environnementales et sociales que le système traditionnel de culture de manioc. Les nouveaux systèmes pourraient être recommandés pour l'intensification de la culture de manioc.

Mots clés : manioc, Gliricidia sepium, soja vert, durabilité, ferralsol.

\section{ABSTRACT}

Study of the economic and environmental sustainability of cassava production on ferralsols Objective: To evaluate the components of the sustainability of cassava cropping systems that rely on alley cropping.

Methodology and results: The study consisted to cultivate improved cassava ( Yavo ) / green soybeans ( Mung Bean) / Gliricidia sepium with a basal dressing ( NPK [ 20:36:36] or chicken manure [10 $\mathrm{t} / \mathrm{ha}$ ] ) compared to the traditional system consisting of a local variety of cassava and peanut intercropping on ferralsols. The sum of the new factors of production allowed a significant increase in the food output. In addition to bean production, 
organic manure and, potentially, fuel wood from G. sepium, the yield of cassava of the introduced variety Yavo was 40 tha compared to 13 tha in the traditional. The plant architecture of the new systems allowed a good soil cover, thus reducing the frequency of weeding, done traditionally by women. Conversely, workload for men increased. The new system was also economically viable.

Conclusion and application of findings: As a whole, the suggested system appears to have a potential to contribute to agronomic, environmental, economic and social sustainability. The new systems could be recommended for the intensification of the cassava crop.

Key words: cassava, Gliricidia sepium, green soya, ferralsol, sustainability.

\section{INTRODUCTION}

La dégradation des sols et la destruction irréversible des terres agricoles progressent à une vitesse alarmante en raison d'un manque de durabilité. La durabilité est considérée comme la capacité des exploitations agricoles à se maintenir dans le temps par obtention de rendements stables adaptés aux faibles capacités financières des producteurs et de revenus réguliers sans nuire de manière irrémédiable à la disponibilité et à la qualité des ressources naturelles. Les recherches sur la production de manioc devraient prendre en compte l'ensemble des aspects agronomiques, environnementaux, économiques et sociaux afin de résoudre durablement les problèmes essentiels. Devant le double enjeu des années à venir: «produire plus» et «disposer d'un aliment de qualité compétitif», le défi face à la baisse de fertilité des sols consisterait à assurer la durabilité des systèmes de culture, condition préalable à la sécurité alimentaire, à la préservation de l'environnement età la lutte contre la pauvreté (Pieri, 1989 ; Peltier, 1990 ; Séne, 1994 ; Young, 1995). La culture de manioc est caractérisée par la culture itinérante sur brûlis où la jachère occupe un rôle important dans la reconstitution de la fertilité des sols. Toutefois, l'abondance des terres en est son corollaire (Kang et al., 1984). La culture itinérante sur brûlis, largement répandue dans les zones tropicales humides, permet

\section{MATERIEL ET METHODES}

Sites d'étude: L'étude s'est déroulée dans quatre villages de la région d'Abengourou; à savoir : Amoriakro $\left(7^{\circ} 098^{\prime} \mathrm{N}, 3^{\circ} 527^{\prime} \mathrm{W}\right)$, Assikasso $\left(7^{\circ} 091^{\prime} \mathrm{N}, 3^{\circ} 236^{\prime} \mathrm{W}\right)$, Duffrebo $\left(7^{\circ} 089^{\prime} \mathrm{N}, 3^{\circ} 448^{\prime} \mathrm{W}\right)$ et Padiégnan $\left(6^{\circ} 979^{\prime} \mathrm{N}\right.$, $3^{\circ} 424^{\prime} \mathrm{W}$ ). Le choix de ces villages pour l'implantation des parcelles expérimentales a été fait en tenant compte des zones d'approvisionnement des usines d'Abidjan. L'expérimentation en milieu paysan, avec deux cycles de d'une part, l'utilisation des nutriments accumulés dans la matière organique mais, d'autre part, engendre des pertes considérables en azote et en soufre (Steiner, 1996). La persistance d'une culture itinérante sur brûlis liée à la très forte croissance démographique en Afrique (environ 3\% par an selon les estimations de l'ONU/OMS [IITA, 2000a]) pose problème et constitue un danger réel pour le développement agricole à long terme. Cela peut signifier une transformation progressive des systèmes de culture itinérante, aujourd'hui largement prédominants, en passant par des systèmes de jachère à courte durée, à une mise en culture permanente sans recours à la jachère (Jean, 1991 ; Hammassa et Coulibaly, 1994 ; IITA, 2000b). Dans ces conditions, il s'avère impératif et urgent de stabiliser l'agriculture en testant des systèmes combinant des variétés améliorées à des plantes de couverture, avec une fumure minérale et/ou organique raisonnée. Ceci contribuera non seulement à l'augmentation de la production alimentaire mais aussi à fixer les populations sur des parcelles données préservant ainsi pour les générations futures, les réserves en forêt actuellement très faibles. La mise en place de tels systèmes contribuerait au développement d'une agriculture durable qui pourrait satisfaire les besoins des générations présentes sans compromettre l'avenir des générations futures.

production de manioc de 18 mois, n'a duré que trois ans ; de 2002 à 2005. L'approche participative a été utilisée afin d'impliquer au même moment tous les acteurs du processus (chercheurs, paysans et vulgarisateurs) du début jusqu'à la fin de l'étude. Le climat est de type subéquatorial (appelé aussi climat attiéen) (Eldin (1971). II comprend deux saisons de pluies (Mars-Juillet et Septembre-Octobre) qui alternent avec deux saisons 
sèches (Novembre-Mars et Août). La pluviométrie oscille entre $1200 \mathrm{~mm}$ et $1800 \mathrm{~mm}$ et la température moyenne annuelle varie de 25 à $28^{\circ} \mathrm{C}$.

Sols : Les parcelles ont été implantées sur des ferralsols dominés par une végétation de Chromolaena odorata. De manière tendancielle, les sols étaient caractérisés par une texture sablo-argileuse (52 à $62 \mathrm{~g} \mathrm{~kg}^{-1}$ de sable), un $\mathrm{pH}$ basique $(7-7,5)$, une faible teneur en carbone organique $\mathrm{C} / \mathrm{N}=10$ à 19 , phosphore assimilable faible autour de $420 \mathrm{mg} \mathrm{kg}^{-1}$ et une capacité CEC faible (4-11 $\left.\mathrm{cmol} \mathrm{kg}^{-1}\right)$. La disponibilité de l'azote pour les végétaux était étroitement liée à la quantité et à la qualité de la matière organique. Le rapport carbone/azote $(\mathrm{C} / \mathrm{N})$, indicateur de la capacité des sols à libérer de l'azote minéral assimilable, était relativement élevé et témoignait d'un humus libérant peu d'azote.

Description des systèmes de culture à base de manioc en compétition : Deux systèmes améliorés ont été mis en compétition avec le système traditionnel. Dans le système traditionnel, la pratique paysanne a consistéà planter simultanément le manioc et l'arachide après la préparation des parcelles sans apport d'intrants. Les activités classiques effectuées comprenaient six opérations, à savoir: le défrichement, le brûlis, le dessouchage, la plantation, le sarclage et la récolte. Deux systèmes améliorés ont été testés en milieu paysan afin de fournir des produits de qualité avec un minimum d'intrants, de minimiser les impacts environnementaux et de conserver le "patrimoine sol» dans toutes ses potentialités. Ces systèmes ont été basés sur la culture en couloirs avec un apport de fumure de fond minérale (200 kg/ha de NPK_10-18-18) ou organique (10 t/ha de fiente de poule). La culture en couloirs comprenait une association de variété améliorée de manioc Yavo/soja vert (Mung bean)/Gliricidia sepium. Le Gliricidia sepium (légumineuse arbustive) a permis d'établir des haies vives à l'intérieur desquelles ont été aménagées quatre lignes de manioc de la variété Yavo. Le soja vert, source importante et relativement peu chère de protéine, a été semé en intercalaire entre les lignes et les plants de manioc afin de favoriser une bonne couverture du sol et une nutrition azotée des plants de manioc. Les activités effectuées comprenaient huit opérations, à savoir : le défrichement, le ramassage des débris végétaux, l'épandage d'engrais, le labour (après épandage de fumure minérale ou organique), la plantation en ligne par piquetage de la superficie réelle de plantation, le sarclage, et la récolte.

Tableau 1 : Répartition sociale des travaux champêtres

\begin{tabular}{|c|c|c|c|c|c|}
\hline \multirow{3}{*}{ Activités agricoles } & \multicolumn{5}{|c|}{ Rôle social joué } \\
\hline & \multicolumn{2}{|c|}{ Hommes } & \multicolumn{2}{|c|}{ Femmes } & \multirow{2}{*}{ Enfants } \\
\hline & Jeunes & Agés & Jeunes & Agés & \\
\hline Défrichement & +++ & ++ & + & - & - \\
\hline Brûlis & ++ & + & t+ & + & - \\
\hline Dessouchage & ++ & + & +++ & + & - \\
\hline Ramassage des débris végétaux & t++ & + & + & + & - \\
\hline Épandage engrais + labour & t++ & ++ & + & - & + \\
\hline Plantation et semis des cultures & + & + & +++ & t+ & + \\
\hline Sarclages ou désherbages & - & - & t++ & ++ & + \\
\hline Récolte soja ou arachide & - & - & t++ & ++ & ++ \\
\hline Récolte manioc & t++ & + & + & - & - \\
\hline
\end{tabular}

- Ne joue aucun rôle, + Rôle secondaire, ++ Rôle important, +++ Rôle très important

Description des opérations agricoles : Le défrichement a été réalisé par les hommes dans les systèmes de culture. Il a consisté à couper le sous-bois à la machette. Le brûlis et le dessouchage ont intervenu dans les systèmes traditionnels. Le brûlis a permis de dégager à l'aide de feu la parcelle encombrée d'adventices coupés et laissés sécher. Le brûlis a été réalisé à $50 \%$ par les deux sexes. Le dessouchage qui a été réalisé par les femmes, a permis de débarrasser des parcelles, des souches laissées après brûlis. Le ramassage des débris végétaux (exécuté par les hommes) et l'épandage engrais minéral ou organique (exécuté par les femmes) + labour (exécuté par les hommes) ont permis respectivement d'éviter le brûlis et d'appliquer une fumure de fond dans le système amélioré (association Yavo/soja vert/Gliricidia sepium). La plantation des cultures a été surtout réalisée par les femmes. Le sarclage ou le désherbage, réalisé majoritairement par les femmes, a permis de tenir la parcelle propre en supprimant les mauvaises herbes. La récolte a commencé avec les cultures à cycle court ( 3 à 4 mois) telles que le soja vert et l'arachide. Cette première récolte a été surtout 
effectuée par les femmes et les jeunes. La deuxième récolte a été celle du manioc. Elle a été réalisée par les hommes. En somme, les travaux qui ont demandé assez d'énergie, à savoir : le défrichement, le ramassage des débris végétaux, le labour manuel et la récolte du manioc, ont été exécutés en majorité par les hommes. Cependant, les travaux nécessitant moins d'effort physique, à savoir : le brûlis, l'épandage de fumure et la plantation des cultures ont été surtout réalisés par les femmes (Tableau 1).

Taux de couverture des systèmes de culture : Le taux de couverture a été mesuré qualitativement à partir d'une évaluation visuelle de l'architecture végétale des parcelles (Tableau 2).

Tableau 2 : Taux de couverture des systèmes de culture

\begin{tabular}{lcc}
\hline \multirow{2}{*}{$\begin{array}{l}\text { Période de mesure après } \\
\text { plantation }\end{array}$} & \multicolumn{2}{c}{ Systèmes de culture } \\
\cline { 2 - 3 } & Systèmes améliorés & Système traditionnel \\
\hline 2 mois & Manioc $($ Yavo $)+$ soja vert & Manioc local + arachide \\
4 mois & Manioc $($ Yavo $)+$ Gliricidia sp & Manioc local \\
6 mois & Manioc (Yavo + Gliricidia sp & Manioc local
\end{tabular}

Évaluation visuelle du couvert végétal des parcelles : 1 = peu dense, 2 = moyennement dense,

$3=$ dense, $4=$ très dense

Calcul de rendements frais en tubercules de manioc : Les récoltes des tubercules de manioc au champ ont été effectuées dans quatre carrés de rendement. L'emplacement des différents carrés a été différent d'une parcelle à une autre. Les dimensions du carré de rendement étaient de $4 \mathrm{~m} \times 4 \mathrm{~m}\left(16 \mathrm{~m}^{2}\right)$ dans les nouveaux systèmes et de $6 \mathrm{~m} \times 6 \mathrm{~m}\left(32 \mathrm{~m}^{2}\right)$ dans le système traditionnel. Ces dimensions ont été choisies en tenant compte de l'écartement des plants de manioc. Le rendement frais ( $\mathbf{R}$ exprimé en $\mathrm{t} / \mathrm{ha}$ ) a été obtenu par la formule suivante :

$$
R(t / h a)=P T F R / S C R
$$

PTFR : Poids Total Frais des Racines tubéreuses SCR : Surface du Carré de Rendement.

Analyse statistique des rendements frais en tubercules de manioc : Les données ont été soumises à l'analyse descriptive avec le logiciel SAS version 8.02 (Statistical Analysis System) (Dervin, 1999 ; Durot, 2002). Les données ont été classées par village, par paysan pilote et par système de culture. L'analyse de variance (Gouet et Philipeau, 1992 ; Dagnelie, 2003) a été faite avec la procédure GLM (General Linear Model) sur les données des deux cycles de production. Les comparaisons des moyennes ont été faites au seuil de $5 \%$. Les différences significatives entre les moyennes ont été déterminées avec le carré moyen ajusté (LSMeans). Calcul du temps de travail (exprimé en hommes-jour par hectare ou $\mathrm{HJ} / \mathrm{ha}$ ): Une enquête a été menée auprès de 60 paysans, soit 15 paysans par village (4 villages : Amoriakro, Assikasso, Duffrebo et Padiégnan). Les questions formulées se rapportaient aux temps de travail des opérations agricoles. La durée moyenne de la journée de travail humain dans les sites d'étude a été de 6 heures ( 1 jour de travail humain $=6$ heures) pour un travail effectué par un actif agricole. Les travaux champêtres débutaient à 8 heures du matin et s'achevaient à 14 heures de l'après midi. Ainsi pour chaque activité agricole, le calcul du temps de travail a été le suivant :

[(Nombre d'heures/6) $x$ nombre de personnes présentes] $/$ Surface $=$ hommes-jour/hectare $(\mathrm{HJ} / \mathrm{ha})$.

Calculs économiques : Le mode de calcul utilisé dans cette section a été inspiré de la méthode de Dufumier (1996) pour caractériser les systèmes de production. Cependant, il a été adapté pour mieux répondre à l'objectif de l'étude. Le calcul a reposé sur la valorisation, aux prix bord champ et du marché et de la totalité des productions de l'unité considérée d'une part; et d'autre part, a permis de comparer les revenus obtenus pour les différents systèmes de production. Ensuite, il a été nécessaire d'affecter, à chaque système de production, les différents postes de dépense correspondants (achats d'intrants, dépenses pour la main-d'œuvre et autres affectations). Ces affectations ont permis de déterminer, pour chaque système de production, le produit brut, le coût de production, la marge brute et le ratio marge brute/coût de production. 
Produit brut : Le produit brut calculé (exprimé en franc CFA ou United States Dollar) a été le produit du rendement commercialisé d'une spéculation et le prix unitaire. Le prix unitaire a désigné le prix bord champ des spéculations. Le prix bord champ des racines fraîches de manioc a été de 20 FCFA (0,04172 dollar), prix fixé par les usines de transformation du manioc. Ceux du soja vert en grain et de l'arachide locale en coque ont été respectivement de 150 FCFA (0,3129 dollar) et 60 FCFA (0,12516 dollar). Toutefois, il n'a pas existé de marché permanent et organisé de boutures de manioc et de chicots de Gliricidia sepium.

Coût de production : Le coût de production a été limité à l'utilisation des intrants (fiente de poule ou engrais NPK) et le travail rémunéré (main d'œuvre contractuelle) pour chaque système de culture. Dans les sites d'étude, la rémunération de la main-d'œuvre externe était fixée à 1000 FCFA (2,086 dollars) par journée de travail. De nombreuses tâches ont été exécutées par contrat. Le

\section{RESULTATS}

Taux de couverture des parcelles : Les résultats ont montré une nette différence entre les systèmes de culture (Tableau 3). Dans l'ensemble, les systèmes améliorés de culture en couloirs (dense) ont bien couvert le sol comparativement au système traditionnel (moyennement dense). Les taux moyens obtenus à 2, 4 et 6 mois après plantation avec les systèmes améliorés ont été respectivement $3,1,3,5$ et 3,6 . Ceux du système traditionnel ont débuté avec un taux de 2,7, ensuite 1,9 et en fin 2,4. Le site d'Assikasso a présenté des résultats relativement plus intéressants que les autres sites.

Tableau 3 : Taux de couverture des parcelles

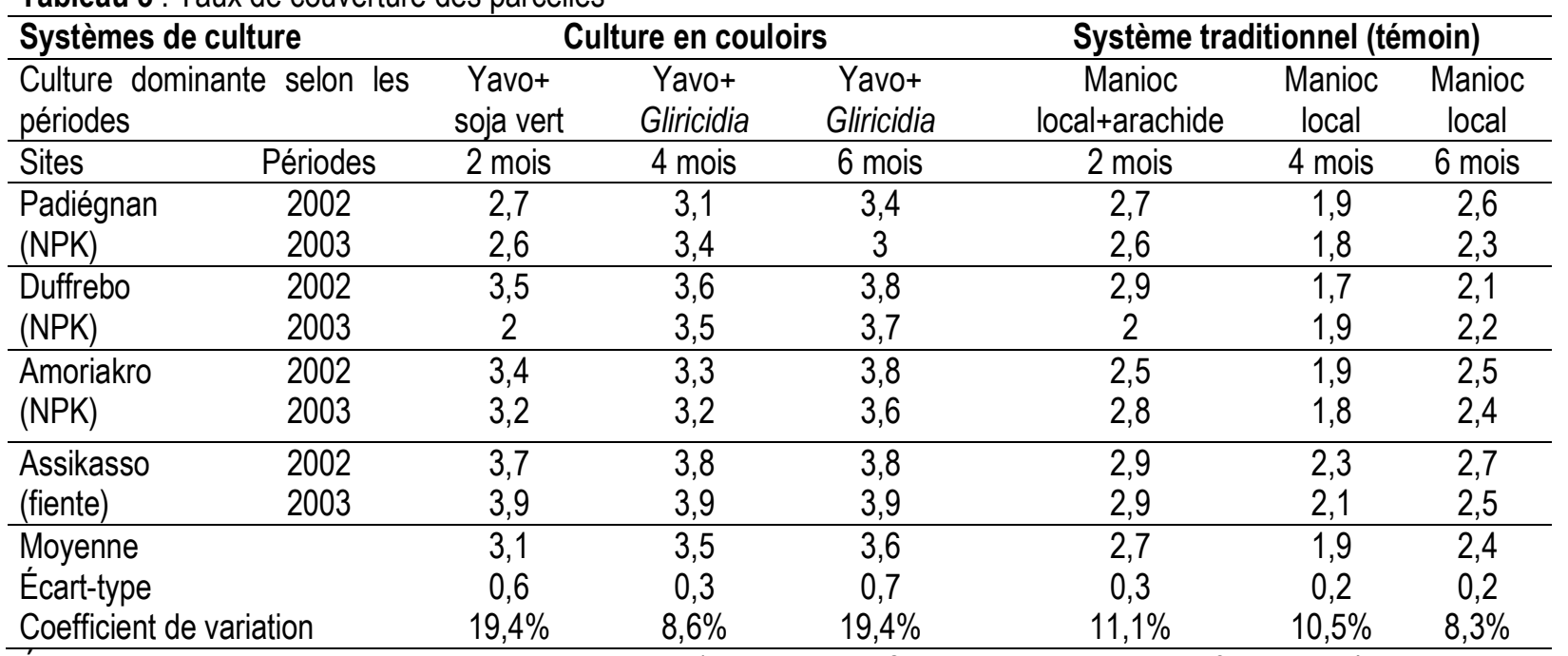

Évaluation visuelle du couvert végétal des parcelles : $1=$ peu dense, $2=$ moyennement dense, $3=$ dense, $4=$ très dense coût a surtout été fonction de l'intensité du travail et seulement secondairement en relation avec la taille de la parcelle.

Marge brute : La marge brute a été la différence entre le produit brut et le coût de production observés au niveau des systèmes de culture de manioc. Elle a permis d'apprécier les coûts et avantages des différentes activités réalisées sur une exploitation.

Ratio marge brute / coût de production : La rentabilité des systèmes de production a été calculée par la méthode du ratio marge brute / coût de production. Le ratio marge brute/coût de production indique ce que rapporte 1 FCFA $(0,002086$ US dollar) investi dans domaine de validité associé au ratio a été défini pour la valeur type 1. Le domaine de validité a permis de rejeter les ratios s'écartant trop de la valeur type 1. Les ratios inférieurs à 1 ont été considérés comme " mauvais ».

Rendement frais en tubercules de manioc à partir des carrés de rendement: Sur les deux cycles de production, le rendement moyen de la variété de manioc Yavo a été de 40 tha et celui des variétés locales de manioc, 13 t/ha (Tableau 4). Le rendement moyen de la variété Yavo a été très hautement supérieur à celui de la variété locale. Cependant, l'effet sites et l'interaction variété $x$ sites ont été non significatifs sur les rendements. Toutefois, la différence de rendement en fin de production pour les trois années d'expérimentation a été statistiquement non significative au niveau des variétés (Tableau 5). l'exploitation. Pour effectuer l'analyse économique, le 


\section{Kouadio et al. J. Appl. Biosci. 2014. Étude de la durabilité économique et environnementale de la production de manioc sur ferralsols}

Tableau 4 : Rendements en racines tubéreuses (t/ha) des variétés de manioc

\begin{tabular}{|c|c|c|c|c|}
\hline \multirow{3}{*}{ Sites et significativité } & \multicolumn{4}{|c|}{ Rendements en tubercules de manioc ( $\mathrm{t} / \mathrm{ha}$ ) } \\
\hline & \multicolumn{2}{|c|}{ Année 2002} & \multicolumn{2}{|c|}{ Année 2003} \\
\hline & Variété Yavo & Variété locale & Variété Yavo & Variété locale \\
\hline Padiégnan (NPK) & 48,9 & 13,1 & 37,6 & 12,3 \\
\hline Duffrebo (NPK) & 41,4 & 11,4 & 39,4 & 13,8 \\
\hline Amoriakro (NPK) & 49,2 & 13 & 33,7 & 13,2 \\
\hline Assikasso (fiente de poule) & 47,4 & 12,8 & 38,9 & 14,5 \\
\hline Moyenne & 46,7 & 12,6 & 37,4 & 13,5 \\
\hline Significativité variété & \multicolumn{2}{|c|}{$<0,0001^{* * *}$} & \multicolumn{2}{|c|}{$<0,0001^{* * *}$} \\
\hline Significativité sites & \multicolumn{2}{|c|}{$\begin{array}{l}0,6171 \mathrm{NS} \\
0,8661 \mathrm{NS}\end{array}$} & \multicolumn{2}{|c|}{$0,2868 \mathrm{NS}$} \\
\hline Significativité variété*sites & \multicolumn{2}{|c|}{$0,8661 \mathrm{NS}$} & \multicolumn{2}{|c|}{$0,7201 \mathrm{NS}$} \\
\hline R-Square $\left(\mathrm{R}^{2}\right)$ & \multicolumn{2}{|c|}{0,85} & \multicolumn{2}{|c|}{0,79} \\
\hline Coefficient de variation (CV) & \multicolumn{2}{|c|}{22,83} & \multicolumn{2}{|c|}{25,54} \\
\hline
\end{tabular}

Tableau 5 : Rendements en racines fraîches de manioc en fin de production au cours des 3 ans d'expérimentations

\begin{tabular}{lccc}
\hline \multirow{2}{*}{ Variétés de manioc } & \multicolumn{3}{c}{ Rendements moyens en racines fraîches (t/ha) } \\
\cline { 2 - 4 } & Cycle 1 (2002) & Cycle 2 (2003) & Différence \\
\hline Manioc Yavo & 46,7 & 37,4 & 9,3 NS \\
Manioc local & 12,6 & 13,5 & 0,9 NS \\
\hline
\end{tabular}

NS = différence non significative

Temps de travail (hommes-jours/ha) : Le Tableau 6 indique les temps de travail des opérations agricoles des systèmes de culture à base de manioc. Le système traditionnel a présenté le temps de travail le plus faible, soit un total de $202 \mathrm{HJ} / \mathrm{ha}$ comparativement à la culture en couloirs (234 à $238 \mathrm{HJ} / \mathrm{ha}$ ). Le besoin en maind'œuvre de la culture en couloirs a été sensiblement le même dans le système avec utilisation de fiente de poule et de NPK soit environ $236 \mathrm{HJ} / \mathrm{ha}$. La quasi-totalité de la main-d'œuvre dans la culture en couloirs (76\%) a été consacrée au défrichement, au ramassage des débris végétaux et à l'épandage d'engrais + labour, soit seulement $6 \%$ pour le sarclage. Cependant, la quasitotalité dans le système traditionnel $(57 \%)$ a été consacrée au défrichement et au sarclage, soit seulement $29 \%$ pour le sarclage. La main-d'œuvre pour le sarclage dans le système traditionnel a été 4 fois plus élevée que celle de la culture en couloirs. La rémunération d'une journée de travail représente ce que gagne le producteur lorsqu'il fait une journée de travail dans son champ. La rémunération a varié d'un système de culture à un autre. Elle a été de 2946 FCFA/HJ (6,15 dollars/HJ) pour la culture en couloirs avec fiente de poule, $2708 \mathrm{FCFA} / \mathrm{HJ}$ ( 5,7 dollars/HJ) pour la culture en couloirs avec NPK et
1466 FCFA/HJ (3,6 dollars/HJ) pour le système traditionnel. La main-d'œuvre étant à 1000 FCFA/jour (2,086 dollars/jour) dans les sites d'étude, un producteur pourra économiser, en une journée de travail sur sa parcelle, 1946 FCFA (4,059356 dollars) pour le système avec fiente de poule, 1708 FCFA (3,6 dollars) pour le système avec NPK et 466 FCFA ( 1 dollar) pour le système traditionnel.

Produit brut: Les résultats individuels de rendements commercialisés de la variété de manioc Yavo ont enregistré de grandes variations. Certains producteurs ont atteint des rendements commercialisés de $50 \mathrm{t} / \mathrm{ha}$. Cependant, la moyenne des rendements commercialisés a été de 37 t/ha. La variété locale de manioc n'a pas présenté de grandes variations au niveau des rendements commercialisés. La variété locale a enregistré des rendements moyens de 13 tha. Le produit brut le plus élevé, 701130 FCFA (1462,6 dollars), a été obtenu au niveau du système avec fiente de poule ; a suivi celui du système avec NPK qui a été de 633630 FCFA (1321,8 dollars) (Tableau 7). Le produit brut du système traditionnel, 296080 FCFA (617,7 dollars), a été inférieur aux deux autres systèmes avec une marge d'environ $44 \%$. 
Tableau 6 : Temps de travail (hommes-jours/ha) des systèmes agricoles

\begin{tabular}{|c|c|c|c|c|c|c|}
\hline \multirow{3}{*}{ Opérations agricoles } & \multicolumn{6}{|c|}{ Temps de travail (hommes-jours/ha ou HJ/ha) } \\
\hline & \multicolumn{2}{|c|}{$\begin{array}{l}\text { Culture en couloirs } \\
\text { (NPK) }\end{array}$} & \multicolumn{2}{|c|}{$\begin{array}{l}\text { Culture en couloirs } \\
\text { (Fiente de poule) }\end{array}$} & \multicolumn{2}{|c|}{$\begin{array}{l}\text { Système traditionnel } \\
\text { (brûlis-sans intrant) }\end{array}$} \\
\hline & $\mathrm{HJ} / \mathrm{ha}$ & $\%$ & $\mathrm{HJ} / \mathrm{ha}$ & $\%$ & $\mathrm{HJ} / \mathrm{ha}$ & $\%$ \\
\hline Défrichement & 65 & 27,8 & 65 & 27,3 & 57 & 28,2 \\
\hline Brûlis & 0 & 0 & 0 & 0 & 12 & 5,9 \\
\hline Essouchement & 0 & 0 & 0 & 0 & 23 & 11,4 \\
\hline Ramassage débris végétaux & 53 & 22,6 & 53 & 22,3 & 0 & 0 \\
\hline Épandage engrais + labour & 59 & 25,2 & 63 & 26,5 & 0 & 0 \\
\hline Plantation des cultures & 24 & 10,3 & 24 & 10,1 & 30 & 14,9 \\
\hline Sarclages & 15 & 6,4 & 15 & 6,3 & 59 & 29,2 \\
\hline Récolte des cultures & 18 & 7,7 & 18 & 7,6 & 21 & 10,4 \\
\hline Taille Gliricidia sepium & n.d & n.d & n.d & n.d & 0 & 0 \\
\hline Main-d'œuvre totale (HJ/ha) & \multicolumn{2}{|c|}{234} & \multicolumn{2}{|c|}{238} & \multicolumn{2}{|c|}{202} \\
\hline Produit brut (FCFA/ha) & \multicolumn{2}{|c|}{633630} & \multicolumn{2}{|c|}{701130} & \multicolumn{2}{|c|}{296080} \\
\hline $\begin{array}{l}\text { Rémunération d'une journée de } \\
\text { travail (FCFA/HJ) }\end{array}$ & \multicolumn{2}{|c|}{2708} & \multicolumn{2}{|c|}{2946} & \multicolumn{2}{|c|}{1466} \\
\hline
\end{tabular}

$0=$ absence de l'opération agricole dans le système de culture

n.d= donnée non disponible, la taille du Gliricidia sepium n'a pas été effectuée au cours des cycles de production.

$\mathrm{HJ} / \mathrm{ha}=$ hommes-jour par hectare

Rémunération d'une journée de travail $(\mathrm{FCFA} / \mathrm{HJ})=$ produit brut $/$ main-d'œuvre totale

Coût d'utilisation des intrants (fumure de fond) : Avec plus d'une centaine de fermes dans la région d'Abengourou, la fiente de poule a été disponible en quantité suffisante. Toutefois, la location de véhicule pour son transport, l'achat des sacs vides de $50 \mathrm{~kg}$, la maind'œuvre pour le remplissage des sacs, le chargement et le déchargement du véhicule ont généré des coûts (Tableau 8). De même, L'achat des sacs de NPK, la location de véhicule pour le transport des sacs, le chargement et le déchargement ont nécessité des sorties de fonds. Le conditionnement de l'engrais chimique NPK a été fait dans des sacs de $50 \mathrm{~kg}$. Le coût de la fertilisation organique a été de 49000 FCFA (102,3 dollars), soit une différence de 5500 FCFA (11,5 dollars) du coût de la fertilisation minérale qui a été de 54500 FCFA (113,7 dollars). Les coûts des intrants ont été nettement en faveur d'une fertilisation organique.

Tableau 7 : Moyenne des produits bruts dégagés par les systèmes de culture

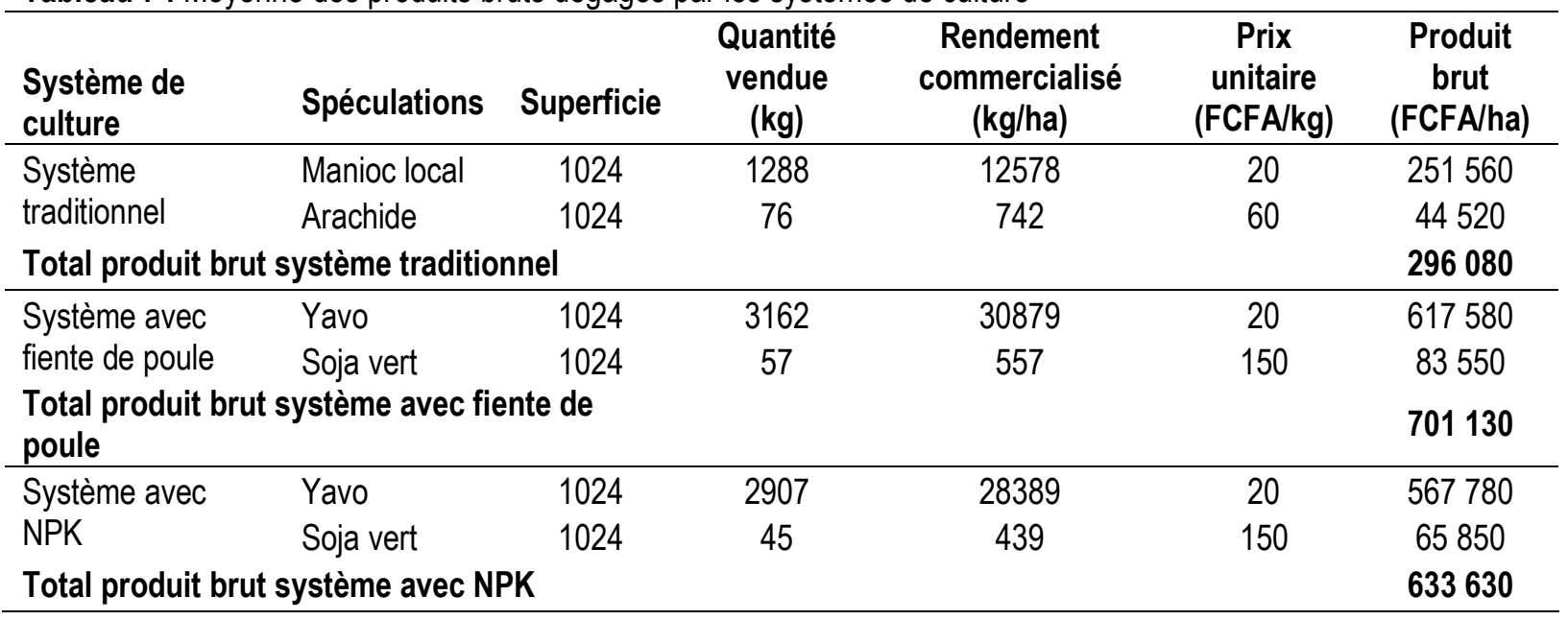


Kouadio et al. J. Appl. Biosci. 2014. Étude de la durabilité économique et environnementale de la production de manioc sur ferralsols

Tableau 8 : Coût d'utilisation des intrants (fumure de fond)

\begin{tabular}{|c|c|c|c|}
\hline \multirow{3}{*}{ Désignations } & \multirow{2}{*}{\multicolumn{2}{|c|}{$\begin{array}{c}\text { Coût en intrants (FCFA) } \\
\text { Culture en couloirs }\end{array}$}} & \multirow{3}{*}{$\begin{array}{l}\begin{array}{l}\text { Système } \\
\text { traditionnel }\end{array} \\
\text { Sans intrant }\end{array}$} \\
\hline & & & \\
\hline & $\begin{array}{c}\text { Fiente de poule } \\
(10 \mathrm{t} / \mathrm{ha})\end{array}$ & NPK (200 kg/ha) & \\
\hline Achat de sacs NPK & - & 48000 & - \\
\hline Achat de sacs vides pour fiente & 15000 & - & - \\
\hline Location de véhicule pour le transport des sacs & 20000 & 5000 & - \\
\hline Main d'œuvre remplissage des sacs de fiente & 4000 & - & - \\
\hline Chargement et déchargement & 10000 & 1500 & - \\
\hline Totaux : & 49000 & 54500 & 0 \\
\hline
\end{tabular}

1. Location de véhicule pour le transport de la fiente : bâchée avec une capacité de $2,5 \mathrm{t}$

2. Location de véhicule pour le transport des sacs d'engrais NPK_10-18-18 de $50 \mathrm{~kg}$ : transport en commun avec frais de bagages (4 sacs de $50 \mathrm{~kg}$ de NPK)

Coûts de production : Le coût de production a été limité à l'utilisation des intrants (fiente de poule ou engrais NPK) et le travail rémunéré (main-d'œuvre contractuelle) pour chaque système de culture. Ainsi, les coûts de production des cultures en couloirs, avec NPK et avec fiente de poule, ont été les plus élevés respectivement 171500 FCFA (357,8 dollars) et 171000 FCFA $(356,706$ dollars) contre 118000 FCFA (246,2 dollars) pour le système traditionnel (Tableau 9). Les coûts de production de la culture en couloirs ont représenté en moyenne plus de $50 \%$ du coût du système traditionnel (plus économe), soit une différence de plus de 50000 FCFA (104,3 dollars). Les coûts les plus importants dans la culture en couloirs ont concerné les postes défrichement (14,6\% aussi bien avec NPK qu'avec fiente de poule), coûts intrants ( $31,8 \%$ pour la culture en couloirs avec NPK et $28,7 \%$ pour la culture en couloirs avec fiente de poule) et épandage engrais + labour (17,5\% pour la culture en couloirs avec NPK et $20,5 \%$ pour la culture en couloirs avec fiente de poule). Par ailleurs, les coûts les plus importants dans le système traditionnel ont concerné les postes défrichement $(16,9 \%)$ et dessouchage $(21,2 \%)$. En revanche, les coûts de production ont été fortement impactés par les postes coûts intrants dans la culture en couloirs et dessouchage dans le système traditionnel.

Tableau 9 : Coûts de production des systèmes de culture en compétition

\begin{tabular}{|c|c|c|c|c|c|c|}
\hline \multirow[t]{2}{*}{ Coûts des activités et intrants } & \multicolumn{2}{|c|}{$\begin{array}{c}\text { Culture en couloirs : } \\
\text { NPK }\end{array}$} & \multicolumn{2}{|c|}{$\begin{array}{l}\text { Culture en couloirs : } \\
\text { fiente de poule }\end{array}$} & \multicolumn{2}{|c|}{$\begin{array}{c}\text { Système } \\
\text { traditionnel }\end{array}$} \\
\hline & FCFA & $\%$ & FCFA & $\%$ & FCFA & $\%$ \\
\hline 1- Défrichement & 25000 & 14,6 & 25000 & 14,6 & 20000 & 16,9 \\
\hline 2- Brûlis & 0 & 0 & 0 & 0 & 5000 & 4,2 \\
\hline 3- Dessouchage & 0 & 0 & 0 & 0 & 25000 & 21,2 \\
\hline 4- Ramassage débris végétaux & 15000 & 8,7 & 15000 & 8,8 & 0 & 0 \\
\hline 5- Coûts intrants & 54500 & 31,8 & 49000 & 28,7 & 0 & 0 \\
\hline 6- Épandage d'engrais + labour & 30000 & 17,5 & 35000 & 20,5 & 0 & 0 \\
\hline 7- Plantation des cultures & 15000 & 8,7 & 15000 & 8,8 & 8000 & 6,8 \\
\hline 8- 1er sarclage & 16000 & 9,3 & 16000 & 9,4 & 16000 & 13,6 \\
\hline 9- $2^{\text {ème }}$ sarclage + récolte arachide & 0 & 0 & 0 & 0 & 6000 & 5 \\
\hline 10- $3^{\text {ème }}$ sarclage & 0 & 0 & 0 & 0 & 12000 & 10,2 \\
\hline 11- 4ème sarclage & 0 & 0 & 0 & 0 & 10000 & 8,5 \\
\hline 12-Récolte soja ou arachide & 6000 & 3,5 & 6000 & 3,5 & 6000 & 5 \\
\hline 13- Récolte manioc & 10000 & 5,8 & 10000 & 5,8 & 10000 & 8,5 \\
\hline Total coûts de production (FCFA) & \multicolumn{2}{|c|}{171500} & \multicolumn{2}{|c|}{171000} & \multicolumn{2}{|c|}{118000} \\
\hline Différence/Système traditionnel & \multicolumn{2}{|c|}{53500} & \multicolumn{2}{|c|}{53000} & \multicolumn{2}{|c|}{0} \\
\hline
\end{tabular}


Marge brute : L'ensemble des résultats a été fortement positif (Tableau 10). II y a eu une grande différence entre le système traditionnel et celles de la culture en couloirs avec NPK ou fiente de poule. Les marges brutes de la culture en couloirs, 462130 FCFA (964,004 dollars) avec
NPK et 530130 FCFA (1105,9 dollars) avec fiente de poule, ont été plus élevées que la marge brute du système traditionnel, 178080 FCFA (371,5 dollars). Avec la culture en couloirs, la marge brute a pu être multipliée par 2,8. Elle a augmenté de $36 \%$.

Tableau 10 : Marge brute des systèmes de culture en compétition

\begin{tabular}{lccc}
\hline Paramètres mesurés & $\begin{array}{c}\text { Culture en couloirs } \\
\text { NPK }\end{array}$ & $\begin{array}{c}\text { Culture en couloirs } \\
\text { fiente de poule }\end{array}$ & Système traditionnel \\
\hline Produit brut (FCFA/ha) & 633630 & 701130 & 296080 \\
Coût de production (FCFA/ha) & 171500 & 171000 & 118000 \\
\hline Marge brute (FCFA) & $\mathbf{+ 4 6 2 1 3 0}$ & $\mathbf{+ 5 3 0 1 3 0}$ & $\mathbf{+ 1 7 8 0 8 0}$ \\
\hline
\end{tabular}

Ratio marge brute/coût de production : Le domaine de validité n'a pas permis de rejeter les ratios calculés. Les ratios ont été supérieurs à 1. II n'y a donc pas eu de mauvais ratios. Les ratios ont varié de 1,51 à 3,10 (Tableau 11). Lorsqu'un paysan a investi un franc CFA $(0,002086$ US dollar) dans la culture en couloirs avec
NPK, cela lui a rapporté 2,69 FCFA (0,0061 dollar) supplémentaires. De même, un franc investi dans la culture en couloirs avec fiente de poule a rapporté une somme supplémentaire de 3,10 FCFA (0,006 dollar) contre 1,51 FCFA (0,004 dollar) supplémentaires pour le système traditionnel.

Tableau 11 : Ratio marge brute/coût de production des systèmes de culture en compétition

\begin{tabular}{lccc}
\hline Paramètres mesurés & $\begin{array}{c}\text { Culture en couloirs } \\
\text { NPK }\end{array}$ & $\begin{array}{c}\text { Culture en couloirs } \\
\text { fiente de poule }\end{array}$ & $\begin{array}{c}\text { Système } \\
\text { traditionnel }\end{array}$ \\
\hline Marge brute (FCFA) & 462130 & 530130 & 178080 \\
Coût de production (FCFA) & 171500 & 171000 & 118000 \\
\hline Ratio marge brute/coût de production & $\mathbf{2 , 6 9}$ & $\mathbf{3 , 1 0}$ & $\mathbf{1 , 5 1}$ \\
\hline \multicolumn{1}{c}{ Domaine de validité : Ratio $>1$} & & &
\end{tabular}

\section{DISCUSSION}

La culture du manioc demande un contrôle efficace des mauvaises herbes, spécialement durant la phase initiale lente de son développement pour des rendements optimums. Ce contrôle pourrait être effectif à travers des pratiques comme cela a été démontré dans la présente étude par l'association culturale, la densité de plantation et l'utilisation de variétés améliorées. L'association culturale manioc amélioré Yavo / soja vert / Gliricidia sepium a bien couvert le sol comparativement au système traditionnel associant manioc local et arachide. La couverture de feuillage du manioc, du soja vert et du Gliricidia sepium a défavorisé la croissance des mauvaises herbes; réduisant ainsi le nombre de sarclages qui était de quatre dans le système traditionnel, à un dans les nouveaux systèmes de culture en couloirs. De façon unanime avec Unamma et Ene (1985), la plantation plus dense protège les cultures des mauvaises herbes et le sol contre l'érosion (Kotschi et al., 1990). Les résultats de rendements de manioc ont montré la très grande variabilité dans la fertilité des sols sous culture. La variété de manioc Yavo associée au soja vert entre des haies de Gliricidia sepium formant des allées de $4 \mathrm{~m}$ de large a enregistré des rendements statistiquement supérieurs à la variété locale, composante principale du système traditionnel. L'innovation de la culture du manioc avec soja, Gliricidia sepium et fumure de fond engrais minéral et/ou fiente de poule a permis d'augmenter le rendement du manioc de 27 tha par rapport au local. Cependant, le rendement moyen de la variété Yavo, obtenu la première année, a été sensiblement supérieur à celui de la deuxième année de culture. La qualité des boutures de manioc ainsi que la pluviométrie plus faible observée la deuxième année (-78\%) pourrait expliquer en partie la différence globale. Par ailleurs, le rendement moyen général en racines tubéreuses fraîches estimé à 40 tha a montré qu'il était possible ici d'obtenir des rendements élevés à l'unité de surface grâce au nouveau système de culture de manioc. Les performances des variétés améliorées de manioc ont été observées dans plusieurs travaux (Kouadio, 2000; El-Sharkawy, 2003). Le rendement faible de la variété locale de manioc est un déterminant majeur de la faible rentabilité du système traditionnel. La main-d'œuvre pour le sarclage dans le système traditionnel a été 4 fois plus élevée que celles de 
la culture en couloirs. Ces résultats coïncident avec ceux de Kang et al. (1984). Pour ces auteurs, dans les petites exploitations, le désherbage a représenté plus de $30 \%$ des exigences en main-d'œuvre. La lutte contre les mauvaises herbes a représenté un coût très important, notamment en temps de travail pour le système traditionnel. Le coût réel du désherbage dans les petites exploitations traditionnelles a été difficile à chiffrer dans la mesure où la main-d'œuvre était essentiellement familiale ou organisée en travaux collectifs villageois, donc non rémunérée. La réduction de la pression en adventices a donc constitué l'un des principaux indicateurs de la durabilité des nouveaux systèmes. Une partie de la maind'œuvre familiale, surtout les femmes, se trouveront libérée grâce à la réduction du nombre de sarclage. Cette main-d'œuvre libre s'orientera vers d'autres activités (activités extra-agricoles, travail salarié.). Ces dernières procureront des revenus additionnels. La valeur de 1,51

\section{CONCLUSION}

La culture en couloirs a assuré une couverture permanente du sol et une augmentation significative du rendement alimentaire. Elle a montré une supériorité économique que le système traditionnel. Le produit brut, la marge brute et le ratio de la culture en couloirs ont été en moyenne 2 fois supérieures que ceux obtenus avec le système traditionnel. La technique de la culture en couloirs du manioc a été d'autant plus bénéfique pour l'exploitant qu'elle a satisfait ses besoins en surfaces cultivables. Toutefois, les coûts de production et le

\section{REFERENCES BIBLIOGRAPHIQUES}

Dagnelie P, 2003. Principes d'expérimentation, planification des expériences et analyses de leurs résultats. Edition Les presses agronomiques de Gembloux. 397p.

Dao D, 2004. Rentabilité comparative de la culture du manioc et analyse de la filière dans la région d'Abengourou. Rapport CSRS, Abidjan, Côte d'Ivoire. $39 \mathrm{p}$.

Dervin C, 1999. Initiation au logiciel statistique SAS sous UNIX. Edition Institut National Agronomique, Paris (France). $63 \mathrm{p}$.

Dufumier M, 1996. Les projets de développement agricoles. Manuel d'expertise. Edition Karthala CTA. Paris, Wageningen. $354 \mathrm{p}$.

Durot C, 2002. S.A.S., Statistical Analysis System. Edition. $54 \mathrm{p}$.

Eldin M, 1971. Le climat. Dans : Le milieu naturel de la Côte d'Ivoire. Mémoires ORSTOM, $n^{\circ}$ 50. pp 73-108.
CFA $(0,003149$ dollar) relevée dans le système traditionnel concorde avec les travaux de Dao (2004) sur la filière manioc dans la région d'Abengourou. Cet auteur a indiqué des ratios de 4 FCFA (0,008344 dollar) pour le cacao, 3 FCFA $(0,006258$ dollar) pour le café et 1,5 FCFA $(0,003129$ dollar) pour le manioc (cas de la culture traditionnelle du manioc). Toutefois, les ratios rencontrés dans la culture en couloirs ont été proches des cultures de rente que sont le cacao et le café. Avec les nouveaux systèmes de culture, le manioc pourrait véritablement être classé comme culture de rente dans la région d'Abengourou. Mais dans un contexte de manque de liquidité, le système traditionnel pourrait rester attrayant malgré sa faible rentabilité. Néanmoins, l'application en milieu réel du système de production améliorée de manioc pourrait être facilitée par la contractualisation ou crédits pour éviter les risques de surproduction.

nombre de main-d'œuvre totale ont été en faveur du système traditionnel. Cependant, la rémunération journalière du travail a été augmentée de facteur 1,93 grâce à la culture en couloirs du manioc. La culture en couloirs pourrait constituer une solution intéressante de remplacement du système traditionnel. Des solutions durables pour atteindre cet objectif devront passer par l'amélioration des termes de l'échange pour la population rurale, l'augmentation des possibilités de marché pour leurs produits, et l'amélioration des infrastructures rurales.

El-Sharkawy MA, 2003. Cassava biology and physiology. Plant Molecular Biology 53: 621-641.

Gouet JP et Philipeau G, 1992. Comment interpréter les résultats d'une analyse de variance? Edition ITCF, Paris (France). $47 \mathrm{p}$.

Hammassa $\mathrm{H}$ et Coulibaly C, 1994. La dynamique foncière face aux exigences du développement durable: le cas de la zone Sud-Mali. In Promotion de systèmes agricoles durables dans les pays d'Afrique soudano-sahélienne. Séminaire régional FAO-CIRAD Dakar Sénégal, 19-14 janvier 1994. pp 137-157.

IITA, 2000a. Politique agricole pour la gestion et l'utilisation durables des ressources naturelles en Afrique. Organisation des Nations Unies pour l'alimentation et l'agriculture. Bureau régional pour l'Afrique, Accra, Ghana. IITA. 96 p.

IITA, 2000b. Plantes de couverture et gestion des ressources en Afrique occidentale. Actes de 
l'atelier organisé par l'IITA et le CIEPCA, du 26 au 29 octobre 1999 à Cotonou, Benin. $316 \mathrm{p}$.

Jean $S, 1991$. Jachères et stratégies foncières. In La jachère en Afrique de l'ouest. Atelier International de Montpellier. ORSTOM éditeurs. Montpellier 2-5 décembre 1991. pp 47-54.

Kang BT, Wilson GF et Lawson TL, 1984. La culture en couloirs : un substitut d'avenir à la culture itinérante. IITA, Ibadan, Nigeria. 22p.

Kotschi J, Water-Byer A, Adelhelm R et Hoesle U, 1990. Agriculture écologique et développement agricole. GTZ. $134 \mathrm{p}$.

Kouadio KKH, 2000. Évaluation des caractères agronomiques des variétés améliorées de manioc (Manihot esculenta CRANTZ) dans la région d'Abengourou (Centre-Est de la Côte d'Ivoire). Mémoire de D.E.A. Université de Cocody, Abidjan, Côte d'Ivoire. 59 p.

Peltier R, 1990. L'arbre dans les terroirs villageois. In Savane d'Afrique, terres fertiles, actes des rencontres internationales, Montpellier France. la Documentation Française, Paris, France. pp. 507-528.

Pieri C, 1989. Fertilité des terres de savane. Bilan de trente années de recherche et de développement agricoles au Sud du Sahara. Paris, Ministère de la coopération et du développement, CIRAD, $444 \mathrm{p}$.

Séne H, 1994. Agro-foresterie : possibilités et limites en tant qu'option de développement durable. In Promotion de systèmes agricoles durables dans les pays d'Afrique soudano-sahélienne. Séminaire régional 10-14 janvier 1994, Dakar, pp 121-133.

Steiner KG, 1996. Causes de la dégradation des sols et approches pour la promotion d'une utilisation durable des sols. Projet pilote « Gestion durable des sols ».GTZ. $58 \mathrm{p}$.

Unamma RPA et Ene LSO, 1985. Évaluation de la nocivité des mauvaises herbes dans la culture du manioc - culture intercalaire du maïs dans la forêt humide du Nigeria. In : Terry, E.R., Doku, E.V., Arene, O.B et Mahungu, N.M. plantes racines tropicales: culture et emplois en Afrique : actes du second symposium triennal de la société internationale pour les plantes racines. Direction Afrique, 14 - 19 août 1983, Douala, Cameroun. Ottawa, Ont., CRDI. pp 6264.

Young A, 1995. L'agroforesterie pour la conservation des sols. ICRAF éditeur. $194 \mathrm{p}$. 